\title{
A SURVEY ADDRESSING THE FUNDAMENTAL MATRIX ESTIMATION PROBLEM
}

\author{
J. Salvi, X. Armangué and J. Pagès \\ University of Girona. \\ Institute of Informatics and Applications. \\ Av. Lluís Santaló, s/n, E-17071 Girona (Spain)
}

\begin{abstract}
Epipolar geometry is a key point in computer vision and the fundamental matrix estimation is the only way to compute it. This article surveys several methods of fundamental matrix estimation which have been classified into linear methods, iterative methods and robust methods. All of these methods have been programmed and their accuracy analysed using real images. A summary, accompanied with experimental results, is given and the code is available in Internet (http: //eia.udg.es/ armangue/research).
\end{abstract}

\section{INTRODUCTION}

The estimation of three-dimensional information in active systems is a crucial problem in computer vision because the camera parameters may change dynamically depending on the scene. In such situations, only epipolar geometry, which is contained in the fundamental matrix, can be computed. Basically, the intrinsic parameters of both cameras and the position and orientation of one in relation to the other can be extracted by using Kruppa equations [1]. Moreover, the fundamental matrix can be used to reduce the matching process among the viewpoints [2], therefore, it is very interesting to develop accurate techniques to compute it.

This article surveys fifteen of the most frequently used techniques in computing the fundamental matrix and is organized as follows. First, a brief introduction of epipolar geometry is presented. Then, all the techniques to estimate $F$ are presented describing their advantages and drawbacks as opposed to previous ones. Section three deals with the experimental results obtained with real images and finally, the article ends with conclusions.

\section{EPIPOLAR GEOMETRY}

Consider a 3D object point $M$ expressed with respect to a world coordinate system $M=\left({ }^{W} X,{ }^{W} Y,{ }^{W} Z, 1\right)^{\mathrm{T}}$ and its $2 \mathrm{D}$ projection $m$ on the image plane in pixels $m=$

\footnotetext{
Work funded by Spanish project CICYT TAP99-0443-C05-01
}

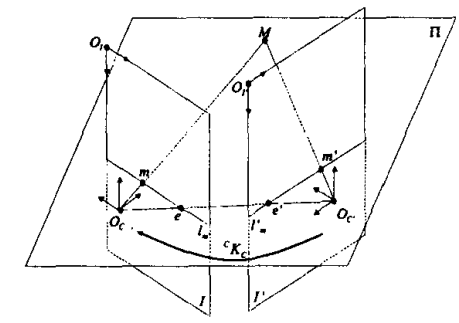

Fig. 1. The geometric relation between two cameras.

$\left({ }^{I} X,{ }^{I} Y\right)^{\mathrm{T}}$. Both points are related with a projective transformation matrix ${ }^{I} \mathrm{P}_{W}$, that is $s m={ }^{I} \mathrm{P}_{W} M$, where $s$ is a scale factor. The ${ }^{I} \mathrm{P}_{W}$ matrix can be broken down into ${ }^{I} \mathrm{P}_{W}={ }^{I} \mathrm{~A}_{C} C_{\mathrm{K}_{W}}$, where ${ }^{I} \mathrm{~A}_{C}$ is a $3 \times 4$ matrix which relates the metric camera coordinate system $C$ located at the focal point $O_{C}$ with the image coordinate system I located at the north-west corner of the image plane in pixels, and $C_{\mathrm{K}_{W}}$ is a $4 \times 4$ matrix, see equation (1), which relates the camera coordinate system $\{\mathrm{C}\}$ with the world coordinate system W.

$$
C_{\mathrm{K} W}=\left(\begin{array}{cc}
C_{\mathrm{R}_{W}} & { }^{C} t_{W} \\
0_{1 \times 3} & 1
\end{array}\right)
$$

Then, the epipolar geometry concerns the relationship between both cameras of a stereoscopic system. Given the object point $M$ and its 2D projections $m$ and $m^{\prime}$ on both image planes, these 3 points define a plane which intersects both image planes at the epipolar lines $l_{m^{\prime}}$ and $l_{m}^{\prime}$ respectively, as shown in Figure 1. Note that the same plane can be computed using both focal points $O_{C}$ and $O_{C^{\prime}}$ and a single 2D projection ( $m$ or $m^{\prime}$ ), which is the principle to reduce the correspondence problem to a single scanning along the epipolar line. Moreover, the intersection of all the epipolar lines define an epipole on both image planes, which can also be extracted by intersecting the line defined from both focal points $O_{C}$ and $O_{C^{\prime}}$ with both image planes. All the epipolar 
geometry is contained in the so called Fundamental matrix F, where $m^{\mathrm{T}} \mathrm{F} m^{\prime}=0$.

The fundamental matrix contains the intrinsic parameters of both cameras and the rigid transformation between both cameras which depends on the camera that has been considered as the origin of the world coordinate system. In this article, the origin of the world coordinate system coincides with the coordinate system of the second camera, located at $O_{C^{\prime}}$, as shown in equation (2).

$$
\mathrm{F}={ }^{I} \mathrm{~A}_{C}{ }^{-\mathrm{T}}\left[{ }^{C} t_{C^{\prime}}\right]_{\times}{ }^{C} \mathrm{R}_{C^{\prime}}{ }^{\prime} \mathrm{A}_{C^{\prime}}{ }^{-1}
$$

\section{ESTIMATING THE FUNDAMENTAL MATRIX}

In the last few years, several methods to estimate the fundamental matrix have been proposed and can be classified into lineal methods and iterative methods. These deal with bad point localization due to noise in image segmentation and robust techniques whitch eliminate the outliers due to false matchings.

\subsection{Linear methods}

The linear method of seven points is based on computing the fundamental matrix by using only seven point correspondences [3]. Due to the homogeneity of the equations, the solution is a set of matrices of the form $F=\alpha F_{1}+(1-\alpha) F_{2}$. Then, by forcing the rank of the matrix to 2 and using the expression det $\left[\alpha F_{1}+(1-\alpha) F_{2}\right\}$, a cubic polynomial is obtained, which has to be solved to obtain $\alpha$ and therefore $F$. The main advantage of this method is that a fundamental matrix can be estimlated by using only seven points, but this fact becomes a drawback when some points are badly located. Moreover, the 7-points method cannot be applied in the presence of redundancy. Hence, it can not be applied using $n$ points if $n>7$.

Another interesting method is 8-points method in witch redundancy of points permits minimizes the error of estimating $\mathrm{F}$. The minimizing equation is $\min _{F} \sum_{i}\left(m_{i}^{\mathrm{T}} F m_{i}^{\prime}\right)^{2}$. The classical method of solving such equation is the leastsquares technique which forces one of the components of $F$ to be the unity [4]. This simplification can be assumed because $\mathrm{F}$ is always defined using a scale factor. Then the system to solve is $f^{\prime}=\left(U^{\prime T} U^{\prime}\right)^{-1} U^{\prime T} c_{9}$ in which $U^{\prime}$ is a matrix containing the first eight columns of $\mathrm{U}, c_{9}$ is the last column of $U$ and $f^{\prime}$ is a vector containing the first eight elements of $f$. Note that the ninth element of $f$ is 1 . A variant of the 8-points method can be applied if the equation is solved by using eigen analysis, also called orthogonal least-squares technique [5]. In this case, $\mathrm{F}$ can be determined from the eigen vector corresponding to the smallest eigen value of $U^{\mathrm{T}} U$. The difference between this method and the classical least-squares resides in the form of calculating the error between correspondences and epipolar lines in which an orthogonal distance to the epipolar line is much more realistic.

The last linear method we surveyed is the analytic method with rank-2 constraint [3] which imposes the rank-2 constraint during the minimization. The matrix $U^{\prime}$ is defined as the composition of the first seven columns of $U$ and $c_{8}$ and $c_{9}$ are defined as the eighth and ninth columns of $U$ respectively so that $\mathrm{F}$ can be computed as

$$
f^{\prime}=-f_{8}\left(\mathrm{U}^{\prime \mathrm{T}} \mathrm{U}^{\prime}\right)^{-1} \mathrm{U}^{\prime \mathrm{T}} c_{8}-f_{9}\left(\mathrm{U}^{\prime}{ }^{\mathrm{T}} \mathrm{U}^{\prime}\right)^{-1} \mathrm{U}^{\prime \mathrm{T}} c_{9}
$$

in which $f^{\prime}$ is the vector containing the first seven elements of $f$, and $f_{8}$ and $f_{9}$ are the eighth and ninth elements of $f$, respectively. In order to obtain the values of $f_{8}$ and $f_{9}$, an $\mathrm{F}$ is computed by using the seven points algorithm. Then, $f$ is computed by selecting from the various pairs of $F$ the one which minimizes $\|f\|=1$. This method provides a rank-2 matrix. However, the analytic method with rank-2 constraint does not improve the results of the previously explained methods to any great extent.

The linear methods are very fast but their accuracy is rather poor in the presence of noise. In order to obtain better results the iterative algorithms have to be considered.

\subsection{Iterative methods}

The iterative methods can be classified into two groups. The first group of techniques is based on minimizing the distances between points and epipolar lines, that is

$$
\min _{\boldsymbol{F}} \sum_{i}\left(d^{2}\left(m_{i}, F m_{i}^{\prime}\right)+d^{2}\left(m_{i}^{\prime}, F m_{i}\right)\right)
$$

A first approach consists of directly applying an iterative method as Newton-Raphson [6]. Another possibility is the iterative linear method [3] which is based on computing the weight value $w_{i}$ equivalent to the epipolar distances by using the previous $\mathrm{F}$ (in the first iteration $w_{i}=1$ ) and then minimize by using least-squares in each iteration. Neither approach imposes the rank-2 constraint. The nonlinear minimization in parameter space [3] can solve this situation. This method is based on parameterizing the fundamental matrix keeping in mind that it must have rank 2 by fixing just one of the multiple parameterizations. The iteration of this method permits computing a better rank- $2 \mathrm{~F}$. However, obtaining a good estimation alone is not enough because the variance of points are not analogous and the least-square technique assumes they are comparable. In order to overcome this drawback, the second group of methods have to be considered.

The second group of methods are based on the gradienttechnique [7]. In this case, the equation to solve is

$$
\min _{\mathbf{F}} \sum_{i}\left(m_{i}^{\mathrm{T}} \mathrm{F} m_{i}^{\prime}\right)^{2} / g_{i}^{2}
$$


in which $g_{i}=\left(l_{1}^{2}+l_{2}^{2}+l_{1}^{\prime 2}+l_{2}^{2}\right)^{1 / 2}$.

The gradient-based technique obtains better results compared to linear methods and the iterative methods of the first group. Although iterative methods are more accurate than linear ones, they are also time consuming and can not eliminate the potential outliers. Hence, robust methods have to be used in the presence of outliers.

\subsection{Robust methods}

This paper surveys three robust methods: M-Estimators, Least-Median-Squares (LMedS) and Random Sampling (RANSAC), which can be used in the presence of either outliers or bad point localization.

The M-estimators [7] try to reduce the effect of outliers by weighting the residual of each point. A lot of different weight functions have been proposed and each one gives a new variant of the M-estimator method. The results obtained are quite good in the presence of outliers but they are rather bad if the points are badly located. The LMedS [3] and $R A N S A C$ [5] techniques are very similar. First, both techniques are based on a random selection of a set of points which are then used to compute $F$ by using a linear method. The difference between these techniques is the way they determining the best $F$. The LMedS calculates the median of distances between points and epipolar lines for each $F$. The chosen fundamental matrix has to minimize such a median. The RANSAC calculates the number of inliers for each $\mathrm{F}$ and the chosen $F$ is the one which maximizes it. Once the outliers are eliminated, the $F$ is recalculated with the aim of obtaining a better approach. Another difference is that LMedS is more restrictive than RANSAC so that it eliminates more points. However, the main constraint of both techniques is their lack of repetitivity due to the aleatory way of selecting the points.

\subsection{Considerations in $\mathrm{F}$ estimation}

Data normalization is a key point in fundamental matrix estimation. It has been proved that the computation should not be applied directly to the raw data in pixels due to potential uncertainties when using by huge numbers. Basically, there are two different methods of data normalization. The first method [3] normalizes the data between $[-1,1]$ The second, proposed by Hartley [8], is based on two transformations. In the first, the points are translated so that their centroid is placed at the origin. In the second, the points are scaled so that the mean of the distances of the points to the origin is $\sqrt{2}$. It has been proved that the method proposed by Hartley gives more accurate results than the previous one.

Another interesting fact is that the estimated $F$ should be a rank-2 matrix in order to model the epipolar geometry with all the epipolar lines intersecting in the epipole.
Although the rank-2 constraint is not imposed in all the surveyed methods, there is a mathematical method which transforms a rank- $n$ square matrix to the closest rank- $(n-1)$ matrix [7]. However, the obtained rank-2 F give worse results because it has not been optimized. In this case, we propose to using any method which imposes a rank- 2 matrix in the computation of $F$ instead of further transforming it.

\section{EXPERIMENTAL RESULTS}

The surveyed methods have been programmed and their accuracy analyzed with synthetic and real data, such as underwater images from the seabed obtained by our underwater robot GARBI. Image points have been normalized by using Hartley [8] explained in section two. Table 1 shows the accuracy of each method as the mean and standard deviation of the distances between points and epipolar lines.

The accuracy of the seven points algorithm extremely depends on the seven points used. The least-squares technique depends inversely on the amount of bad-located points, obtaining usually better results by increasing the amount of points. The eigen analysis is the linear method that obtains the best results because an orthogonal least-squares minimization is more realistic than the classic least-squares. However, all these methods obtain a rank-3 matrix, which means that the epipolar geometry is not properly modeled.

The analytic method with rank-2 constraint obtains a rank-2 fundamental matrix. However, the distances between points and epipolar lines are worse than in the linear methods. The iterative linear method improves considerably the least-squares technique but can not cope with the outliers problem. The iterative Newton-Raphson algorithm obtains even better results than the previous method if there is no outliers present. Although the nonlinear minimization in parameter space obtains also a rank-2 matrix, but his computational cost is very high. The eighth and ninth methods are two versions of the gradient-based method using leastsquares and orthogonal least-squares, respectively. Both methods obtain better results than their equivalent linear methods. Furthermore, the eigen analysis, once again, obtains better results than the other linear methods. Although some of these methods obtain a rank- 2 matrix, they can not cope with outliers.

The last surveyed methods are known as "robust", which means they might detect and remove the outliers and compute the fundamental matrix using only the inliers. Three versions of the M-estimators have been programmed using least-squares, eigen analysis and the method proposed by Torr [5], respectively. The three methods use a linear initial guess and they become really dependent on the linear method used to estimate it. The following two methods are two versions of LMedS using again least-squares and eigen analysis, respectively. Although the accuracy of 
Table 1. Methods Implemented with mean and std. of error: 1.- seven points; 2.- least-squares (LS) 3.- orthogonal LS; 4.- rank2 constraint; 5.- iterative lineal using LS; 6.- iterative Newton-Raphson using LS; 7.- minimization in parameter space using eigen; 8 . gradient using LS; 9.- gradient using eigen; 10.- M-Estimator using LS; 11.- M-Estimator using eigen; 12.- M-Estimator proposed by Torr; 13.- LMedS using LS; 14.- LMedS using eigen; 15.- RANSAC using eigen.

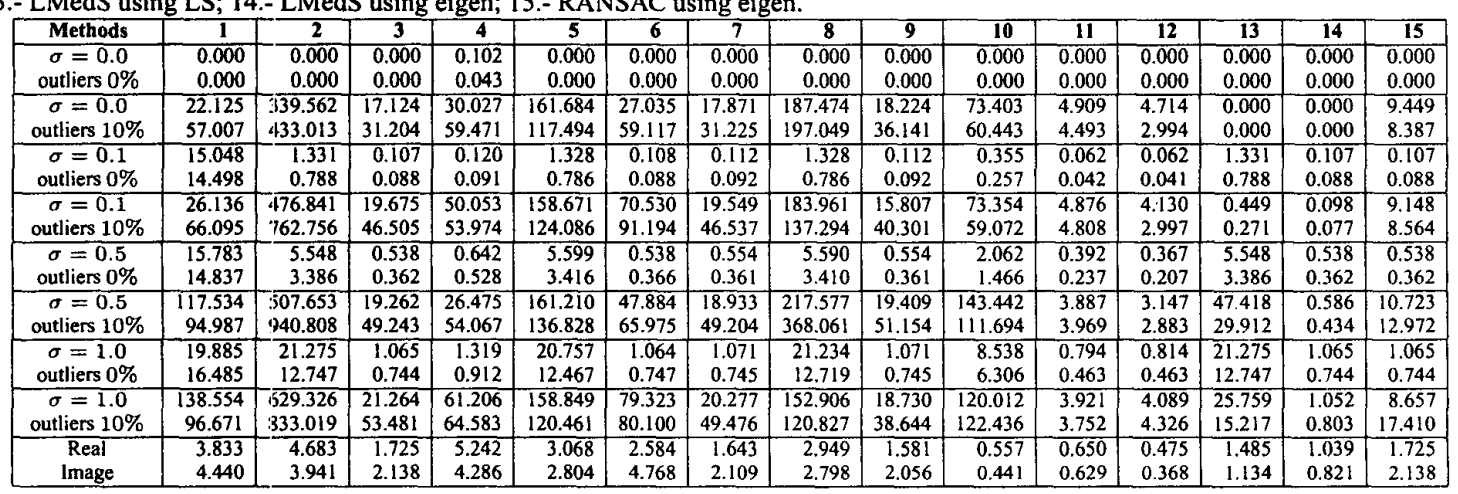

$L M e d S$ seems worse than the one given by $M$-estimators, $L M e d S$ removes the outliers much more correctly since the epipolar geometry is better modeled. The RANSAC is the last surveyed method, which does not obtain better results than $L M e d S$ with eigen analysis because the method is too permissive selecting the outliers.

\section{CONCLUSIONS}

The objective of this article is a comparative survey of fifteen of the most frequently used methods in fundamental matrix estimation. The different methods have been programmed and their accuracy analyzed with real images. Experimental results show that: a) linear methods are quite good if the points are well located in the image and the correspondence problem previously solved; b) iterative methods can cope with some gaussian noise in the localization of points, but they become quite inefficient in the presence of outliers; c) robust methods can cope with both discrepancy in the localization of points and false matchings.

The experimental results point out that the orthogonal least-squares using eigen analysis gives better results than the classic least-squares technique of minimization. Moreover, a rank-2 method is preferred because it models the epipolar geometry with. all the epipolar lines intersecting at the epipole. Finally, experimental results show that the corresponding points have to be normalized and the best results using this sort of method have been obtained by using the method proposed by Hartley [7]. In conclusion, the best results were obtained with the $L M e d S$ method forcing the matrix to be rank- 2 once the outliers have been removed.

\section{REFERENCES}

[1] Richard I. Hartley, "Kruppa's equations derived from the fundamental matrix.," Pattern Analysis and Machine Intelligence, vol. 19, no. 2, pp. 133-135, 1997.

[2] Zhengyou Zhang, "The matching problem: The state of the art," Tech. Rep. 2146, Institut National de Recherche en Informatique et en Automatique, 1993.

[3] Zhengyou Zhang, "Determining the epipolar geometry and its uncertainty: A review," Tech. Rep. 2927, Institut National de Recherche en Informatique et en Automatique, July 1996.

[4] Quan-Tuan Luong and Olivier D. Faugeras, "The fundamental matrix: Theory, algorithms, and stability analysis," International Journal of Computer Vision, vol. 17, no. 1, pp. 43-75, January 1996.

[5] P. H. S. Torr and D. W. Murray, "The developement and comparision of robust methods for estimating the fundamental matrix," International Journal Computer Vision, vol. 24, no. 3, pp. 271-300, September 1997.

[6] Joaquim Salvi, An Approach to Coded Structured Light to Obtain Three Dimensional Information, Ph.D. thesis, Universitat de Girona, Departament d'Electrònica, Informàtica i Automàtica, 1997.

[7] Richard Hartley and Andrew Zisserman, Multiple View Geometry in Computer Vision, Cambridge University Press, 2000.

[8] Richard Hartley, "In defence of the 8-point algorithm," in Proceedings of the 5th International Conference on Computer Vision, Boston, 1995, pp. 1064-1070, IEEE Computer Society Press. 